\title{
De novo resveratrol production through modular engineering of an Escherichia coli-Saccharomyces cerevisiae co-culture
}

\author{
Shuo-Fu Yuan ${ }^{1}$, Xiunan $\mathrm{Yi}^{1}$, Trevor G. Johnston ${ }^{2}$ and Hal S. Alper ${ }^{1,3^{*}}$
}

\begin{abstract}
Background: Resveratrol is a plant secondary metabolite with diverse, potential health-promoting benefits. Due to its nutraceutical merit, bioproduction of resveratrol via microbial engineering has gained increasing attention and provides an alternative to unsustainable chemical synthesis and straight extraction from plants. However, many studies on microbial resveratrol production were implemented with the addition of water-insoluble phenylalanine or tyrosine-based precursors to the medium, limiting in the sustainable development of bioproduction.
\end{abstract}

Results: Here we present a novel coculture platform where two distinct metabolic background species were modularly engineered for the combined total and de novo biosynthesis of resveratrol. In this scenario, the upstream Escherichia coli module is capable of excreting $p$-coumaric acid into the surrounding culture media through constitutive overexpression of codon-optimized tyrosine ammonia lyase from Trichosporon cutaneum (TAL), feedback-inhibitionresistant 3-deoxy-d-arabinoheptulosonate-7-phosphate synthase ( $a{ }^{\prime}\left(G^{f b r}\right)$ and chorismate mutase/prephenate dehydrogenase $\left(t y r A^{f b r}\right)$ in a transcriptional regulator tyrR knockout strain. Next, to enhance the precursor malonyl-CoA

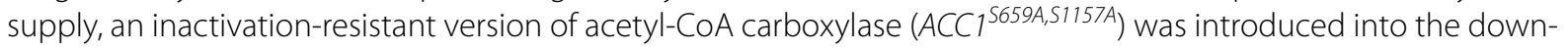
stream Saccharomyces cerevisiae module constitutively expressing codon-optimized 4-coumarate-CoA ligase from Arabidopsis thaliana (4CL) and resveratrol synthase from Vitis vinifera (STS), and thus further improve the conversion of $p$-coumaric acid-to-resveratrol. Upon optimization of the initial inoculation ratio of two populations, fermentation temperature, and culture time, this co-culture system yielded $28.5 \mathrm{mg} / \mathrm{L}$ resveratrol from glucose in flasks. In further optimization by increasing initial net cells density at a test tube scale, a final resveratrol titer of $36 \mathrm{mg} / \mathrm{L}$ was achieved.

Conclusions: This is first study that demonstrates the use of a synthetic E. coli-S. cerevisiae consortium for de novo resveratrol biosynthesis, which highlights its potential for production of other $p$-coumaric-acid or resveratrol derived biochemicals.

Keywords: Resveratrol, Modular metabolic engineering, Synthetic co-culture system

\section{Background}

Resveratrol is a plant-derived stilbenoid compound, commonly found in grape extract and red wine, that is touted for bioactive properties including antioxidant,

*Correspondence: halper@che.utexas.edu

${ }^{3}$ McKetta Department of Chemical Engineering, The University of Texas at Austin, 200 E Dean Keeton St. Stop C0400, Austin, TX 78712, USA

Full list of author information is available at the end of the article anti-inflammatory, anti-tumor, cardio- and neuro-protective properties [1-4]. Given the increasing interest in these health-related benefits, the global market for resveratrol is expected to almost double in the next 6 years from US\$ 58 million (in 2020) to US\$ 99.4 million by 2026 [5]. To meet this growing demand and bypass ecounfriendly chemical syntheses and direct extraction from natural sources [6-8], there have been numerous metabolic engineering approaches for microbial resveratrol 
production [9-14]. Biochemically, resveratrol synthesis requires 4-coumaroyl-CoA whose biosynthesis starts with the conversion of phenylalanine and tyrosine into the phenylpropanoid acids cinnamic acid and $p$-coumaric acid, respectively [15]. These reactions are catalyzed by phenylalanine ammonia lyase (PAL) and tyrosine ammonia lyase (TAL) enzymes with some promiscuous crossreactivity known to be present [16]. Cinnamic acid can be further hydroxylated by a cytochrome P-450-dependent cinnamate-4-hydroxylase $(\mathrm{C} 4 \mathrm{H})$ to form $p$-coumaric acid. In both routes, the resulting $p$-coumaric acid is subsequently biotransformed to 4-coumaroyl-CoA by
4-coumaroyl-CoA ligase (4CL) and then finally into resveratrol by the sequential condensations with malonylCoA catalyzed by a stilbene synthase (STS) [17] (Fig. 1).

Using the approaches of metabolic engineering, common host microorganisms including $E$. coli and S. cerevisiae as well as a variety of non-conventional hosts have been extensively engineered for resveratrol bioproduction [9-14, 18-20]. However, most efforts do not describe purely de novo production and thus require the supplementation of relatively expensive and low-watersolubility substrates such as $p$-coumaric acid or aromatic amino acids $[15,17]$. One standout report for de novo

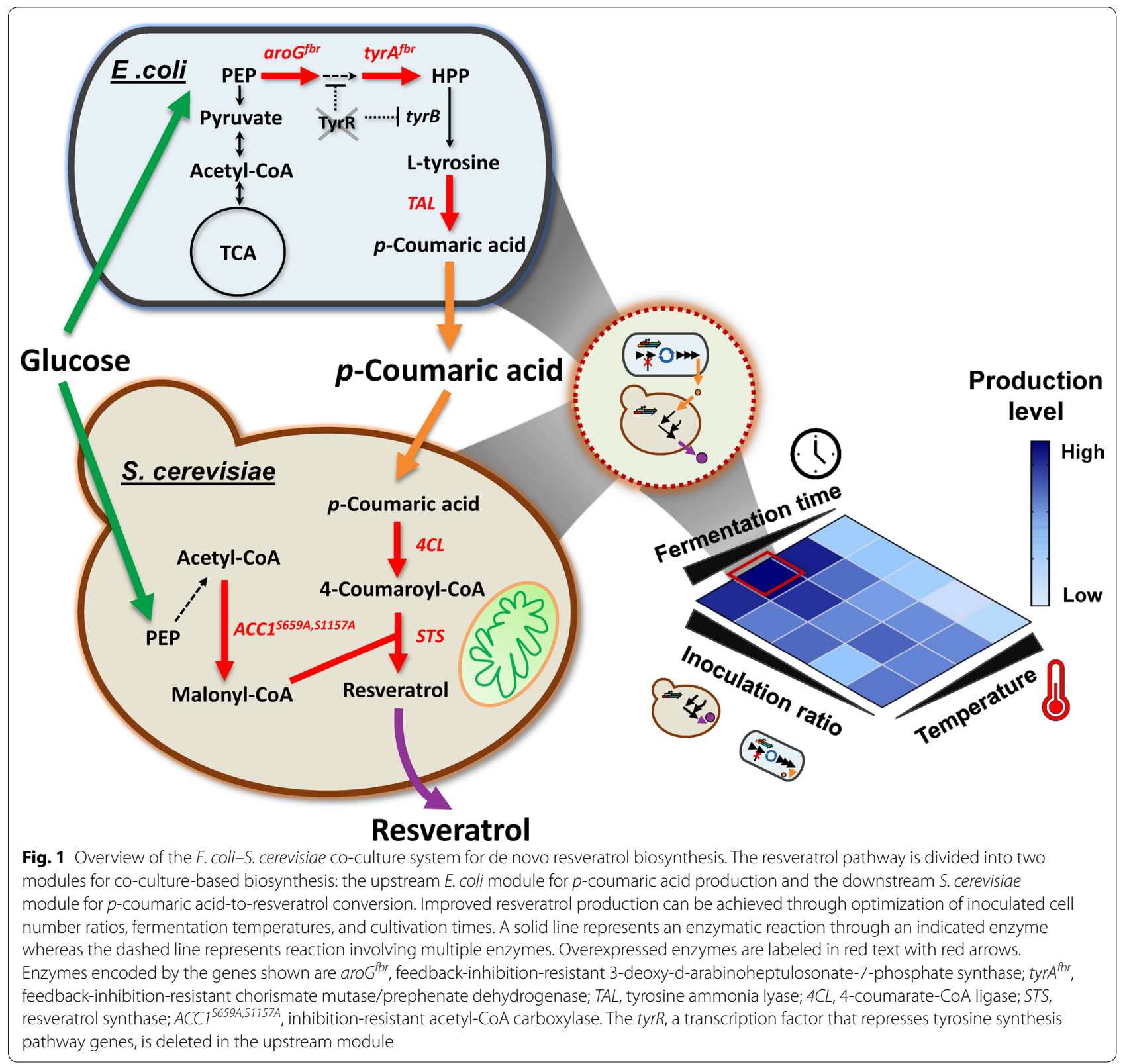


production from glucose and ethanol was achieved in S. cerevisiae CEN.PK102-5B $[10,11]$ whereby extensive engineering of the tyrosine pathway along with complementation of resveratrol biosynthetic genes (TAL from $H$. aurantiacus, $4 C L$ from $A$. thaliana and STS from V. vinifera) led to a resveratrol titer of 416 and $531 \mathrm{mg} / \mathrm{L}$ from glucose and ethanol, respectively, in fed-batch fermentation [10]. Further improvements were made by using the phenylalanine pathway to achieve a final titer of 812 and $755 \mathrm{mg} / \mathrm{L}$ resveratrol from glucose and ethanol feed, respectively, in fed-batch mode [11].

Despite these decent titers, S. cerevisiae does not have a very strong innate flux toward aromatic amino acids and derived products. In circumstances wherein metabolic potential is restricted, co-culture strategies have been explored. In this regard, co-culture strategies can improve production by dividing complex and extensive pathways into individual modules, thus reducing the metabolic burden of each independent microbial strain and leveraging the innate metabolic potential of each host [21-24]. In doing so, this strategy enables parallel construction of optimized metabolic pathways and utilizes cross-feeding at key metabolite nodes [25, 26].

To date, there are only two published studies utilizing microbial co-culture for the production of resveratrol. The first case demonstrated an E. coli-E. coli co-culture using W3110s to produce resveratrol from glycerol [27]. In this scheme, the first $E$. coli module was engineered to produce $p$-coumaric acid through the overexpression of TAL from Rhodothorula glutinis, aroG $G^{f b r}$, and $t k t A$ in the background of a pheA knockout mutant. The second $E$. coli module utilized the $p$-coumaric acid and converted it into resveratrol via overexpression of heterologous genes $4 C L$ from Streptomyces coelicolor A2 and STS from Vitis vinifera. The resulting co-culture system led to a final titer of $22.6 \mathrm{mg} / \mathrm{L}$ resveratrol in a bioreactor while still requiring IPTG induction. In the second case, another E. coli-E. coli co-culture (this time using MG1655 strain background) produced $55.7 \mathrm{mg} / \mathrm{L}$ resveratrol from glucose [14]. In this scheme, the $p$-coumaric acid-producing strain was generated through the introduction of $a r o G^{f b r}$, tyr $A^{f b r}$ and $R$. glutinis TAL into a tyrR and pgi (encoding the first-step enzyme of the EMP pathway) knockout background. The second strain produced resveratrol through heterologous overexpression of C. glutamicum acc, Petroselinum crispum 4CL and Arachis hypogaea STS in conjunction with a $z w f$ deletion. As with the first case, this co-culture leveraged the $\mathrm{P}_{\text {Lteto-1 }}$ promoter and thus requires induction by an expensive inducer such as doxycycline.

Based on these prior results, no study has used a coculture system for resveratrol production without the need for expensive inducers and with distinct organisms.
The only instances described above used an E. coli-E. coli co-culture strategy that does not leverage distinct metabolic capacities. In this work, we developed a unique consortium utilizing two metabolically distinct microorganisms, E. coli and S. cerevisiae, for de novo resveratrol production from glucose. In doing so, we utilize a direct, one-step route for conversion of tyrosine into $p$-coumaric acid through heterologous overexpression of a tyrosine ammonia lyase from $T$. cutaneum $(T A L)$ in a E. coli tyrosine overproducer [28] (designated as the upstream module). In the second host, we chose S. cerevisiae to better express plant-derived resveratrol biosynthetic enzymes due to its ability for proper protein folding and posttranslational modification. In this regard, we rewired this host to convert $p$-coumaric acid into resveratrol via chromosomally integrated expression of $A C C 1^{S 659 A, S 1157 A}$, $A$. thaliana $4 C L$ and $V$. vinifera STS (designated as the downstream module). Through a series of optimization for media composition, inoculation ratios, fermentation temperatures, and initial net cells density, we obtained $36 \mathrm{mg} / \mathrm{L}$ resveratrol in a purely de novo fashion without the need for supplementation of expensive inducers or precursors. The platform described here thus enables the first demonstration of a synthetic E. coli-S. cerevisiae consortium for de novo resveratrol production.

\section{Results and discussion}

\section{Escherichia coli-S. cerevisiae co-culture design} and construction

In this work, we chose to select an E. coli-S. cerevisiae co-culture to take advantage of these two distinct organisms. As stated above, the downstream enzymes in this pathway are more compatible with the eukaryotic environment of S. cerevisiae. Additionally, previous reports have demonstrated that 4-coumaroyl-CoA can inhibit the activity of the upstream TAL enzyme [29]. As a result, separating the expression of TAL and 4CL enzymes would bypass an undesired feedback-inhibitory crosstalk within the same host. The basic design for this synthetic co-culture is shown in Fig. 1.

We constructed the upstream module in E. coli by taking advantage of a more robust metabolic potential for aromatic amino acid pathways. To do so, we created a tyrosine overproducer strain of E. coli BL21(DE3) consisting of a tyrR knockout along with constitutive overexpression of feedback-inhibition-resistant versions of $\operatorname{aro} G^{f b r}$ and $t y r A^{f b r}$ [28]. In this background, we then redirected metabolic flow from intracellular tyrosine pools to $p$-coumaric acid by expressing a heterologous, codon-optimized T. cutaneum TAL gene (Additional file 1: Table S2) [30] under the control of a constitutive promoter with a strong ribosomal binding site. The resulting strain (named eBL0430T) exhibited a high titer 
of $p$-coumaric acid $(414 \mathrm{mg} / \mathrm{L})$ with good biomass production, especially compared to a strain with lower gene expression and production level (named strain eBL0432T producing $122 \mathrm{mg} / \mathrm{L}$, Additional file 1: Fig. S1). As a result, this strain was selected for use in the co-culture.

To establish the downstream module for $p$-coumaric acid-to-resveratrol conversion in S. cerevisiae, we chromosomally integrated constitutive expression cassettes for codon-optimized $4 C L$ and STS (Additional file 1: Table S2) [10] into the BY4741 strain. To increase the supply of intracellular malonyl-CoA, we subsequently integrated a feedback resistant mutant, $A C C 1^{S 659 A, S 1157 A}$ [10], into this strain to yield a final strain (named sBY11). This resulting strain exhibited bioconversion of $p$-coumaric acid into resveratrol and thus was selected for use in the co-culture.

Once these two hosts were constructed, we evaluated the synthetic co-culture's capacity to product resveratrol in a de novo manner. Specifically, we tested production in a minimal medium (MM1) using an inoculation ratio of $1: 1$ with a middle-ground co-cultivated temperature of $33.5^{\circ} \mathrm{C}$ (Fig. 2a-c). In this condition, a maximum resveratrol titer of $5.3 \mathrm{mg} / \mathrm{L}$ was achieved at 48 -h timepoint (with a yield of $0.26 \mathrm{mg}$ resveratrol/g glucose) (Fig. 2a), however, a higher amount of $p$-coumaric acid $(30.2 \mathrm{mg} / \mathrm{L})$ was observed in this condition (Fig. 2b). Moreover, the growth of this co-culture (Fig. 2c) indicated that the conversion issues could be due to the poor co-culture growth in this minimal media formulation.

Previous studies have demonstrated significantly improved consortia performance and biomass formation with the addition of some nutrients. As examples, increasing the concentration of yeast extract from $1 \mathrm{~g} / \mathrm{L}$ to $2 \mathrm{~g} / \mathrm{L}$ in an $E$. coli-E. coli co-culture resulted in a nearly 136 -fold increase in monolignol $p$-coumaryl alcohol production [24]. Additionally, nutrient optimization in an E. coli-S. cerevisiae consortium led to a 3.1-fold increase in naringenin biosynthesis [31]. With these results as context, we investigated a nutrient-rich media formulation (RM1) to test its effect on co-culture performance (Fig. $2 \mathrm{~d}-\mathrm{f}$ ). In doing so, we repeated the $E$. coli eBL0430T-S. cerevisiae sBY11 co-culture at $33.5^{\circ} \mathrm{C}$ with an inoculation ratio of $1: 1$. In this case, the consortia was able to produce more resveratrol $(7.8 \mathrm{mg} / \mathrm{L}$ vs. $5.3 \mathrm{mg} / \mathrm{L})$ and accumulated less $p$-coumaric acid $(9.3 \mathrm{mg} / \mathrm{L}$ vs. $30.2 \mathrm{mg} / \mathrm{L}$ ) when compared with that of MM1 medium used above (compare Fig. 2d, e with Fig. 2a, b). Moreover, under this culture condition, resveratrol was gradually produced over time with concomitant decrease in $p$-coumaric acid, thus implying that the downstream yeast module was more apt to convert this substrate in this media condition. Furthermore, biomass accumulation was enhanced in this complex RM1 medium compared with the defined medium above (comparing Fig. $2 \mathrm{f}$ to c). As a result, the RM1 medium was used for the following experiments.

\section{Investigating the impacts of inoculation ratio and fermentation temperature on resveratrol biosynthesis} Maintaining a stable and robust composition of organisms within a co-culture is essential for efficient biochemical production [26]. In this case, we are utilizing two organisms with different optimal temperatures for growth thus implying culture temperature as an important parameter in co-culture performance. To this end, we explored the impacts of varying fermentation temperature $\left(25,30,33.5\right.$ and $\left.37^{\circ} \mathrm{C}\right)$, time $(20,48$, and $72 \mathrm{~h})$, and initial inoculation ratio of engineered strains (100:1, 10:1, 1:1, 1:10 to 1:100) in a large-scale test tube system (Fig. 3 and Additional file 1: Fig. S3).

In general, cultivation at the two higher temperatures $\left(33.5\right.$ and $\left.37^{\circ} \mathrm{C}\right)$ displayed higher productivities and titers of resveratrol during the early-middle stage of fermentation (20 and $48 \mathrm{~h}$ ) compared with the lower temperature range of $25-30{ }^{\circ} \mathrm{C}$ (Table 1 and Fig. 3a). Specifically, the synthetic consortia incubated at these high temperatures $\left(33.5-37{ }^{\circ} \mathrm{C}\right)$ exhibited averaged resveratrol titers that were up to 15.74 -fold higher than those at lower temperatures (Additional file 1: Fig. S3a, b). Additionally, these elevated temperature cultures also produced nearly 4-fold less $p$-coumaric acid than that at relatively low temperatures $\left(25\right.$ and $30{ }^{\circ} \mathrm{C}$ ) (Fig. $3 \mathrm{~b}$ and Additional file 1: Fig. S3d, e). These results demonstrated that a higher cultivation temperature range of $33.5-37{ }^{\circ} \mathrm{C}$ resulted in improved resveratrol productivity from the consortia at the early-to-middle phase of fermentation, thus leading to a faster conversion of $p$-coumaric acid into resveratrol.

Despite these results at early-to-middle range, the averaged final titer of the conditions incubated at $37^{\circ} \mathrm{C}$ across a range of inoculation ratio (100:1-1:100; with $3.01 \mathrm{mg} / \mathrm{L}$ resveratrol) was lower than that of $30^{\circ} \mathrm{C}(4.42 \mathrm{mg} / \mathrm{L})$ and $33.5^{\circ} \mathrm{C}(6.08 \mathrm{mg} / \mathrm{L})$. This result indicates that the consortia's metabolic activity at $37^{\circ} \mathrm{C}$ suffered in the later phase of fermentation (48-72 h) compared with the lower temperatures, suggesting $33.5{ }^{\circ} \mathrm{C}$ was a more favorable fermentation temperature for the synthetic consortia when operating in batch culture mode.

As expected, the final resveratrol content was significantly influenced by the inoculation ratio (tested from 100:1 to $1: 100)$ across a range of temperatures $\left(25-37^{\circ} \mathrm{C}\right)$ and over time (20-72 h) (Fig. 3a and Additional file 1: Fig. S3a-c). The averaged resveratrol titers of conditions with higher inoculated yeast-to-E. coli ratios (100:1 and 10:1) were between 1.21 and 7.70-fold higher than the conditions with lower inoculation ratios (1:10 and 1:100) (Fig. 3a). These results highlight that the downstream 


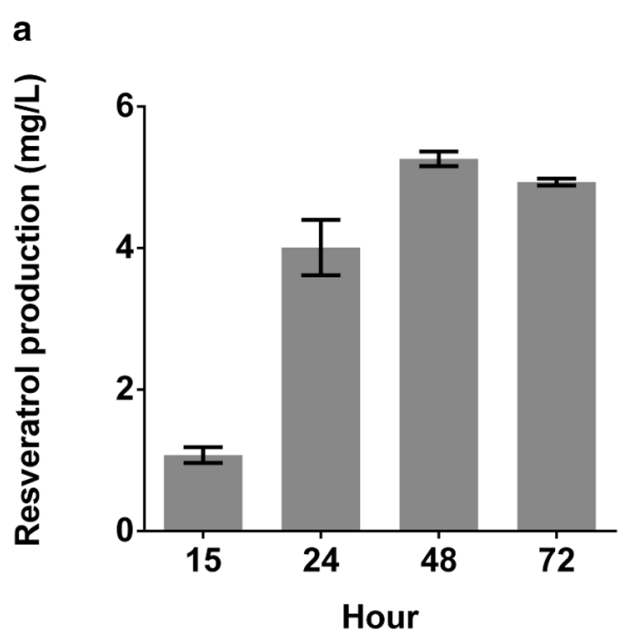

\section{d}
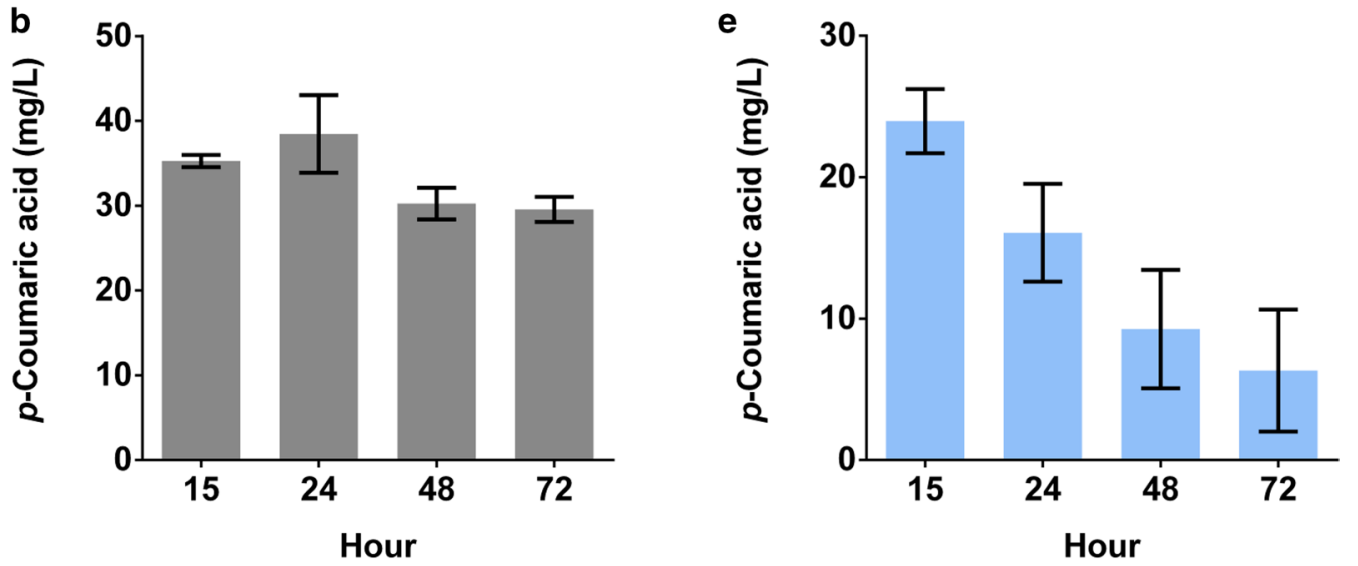

C

$\mathbf{f}$
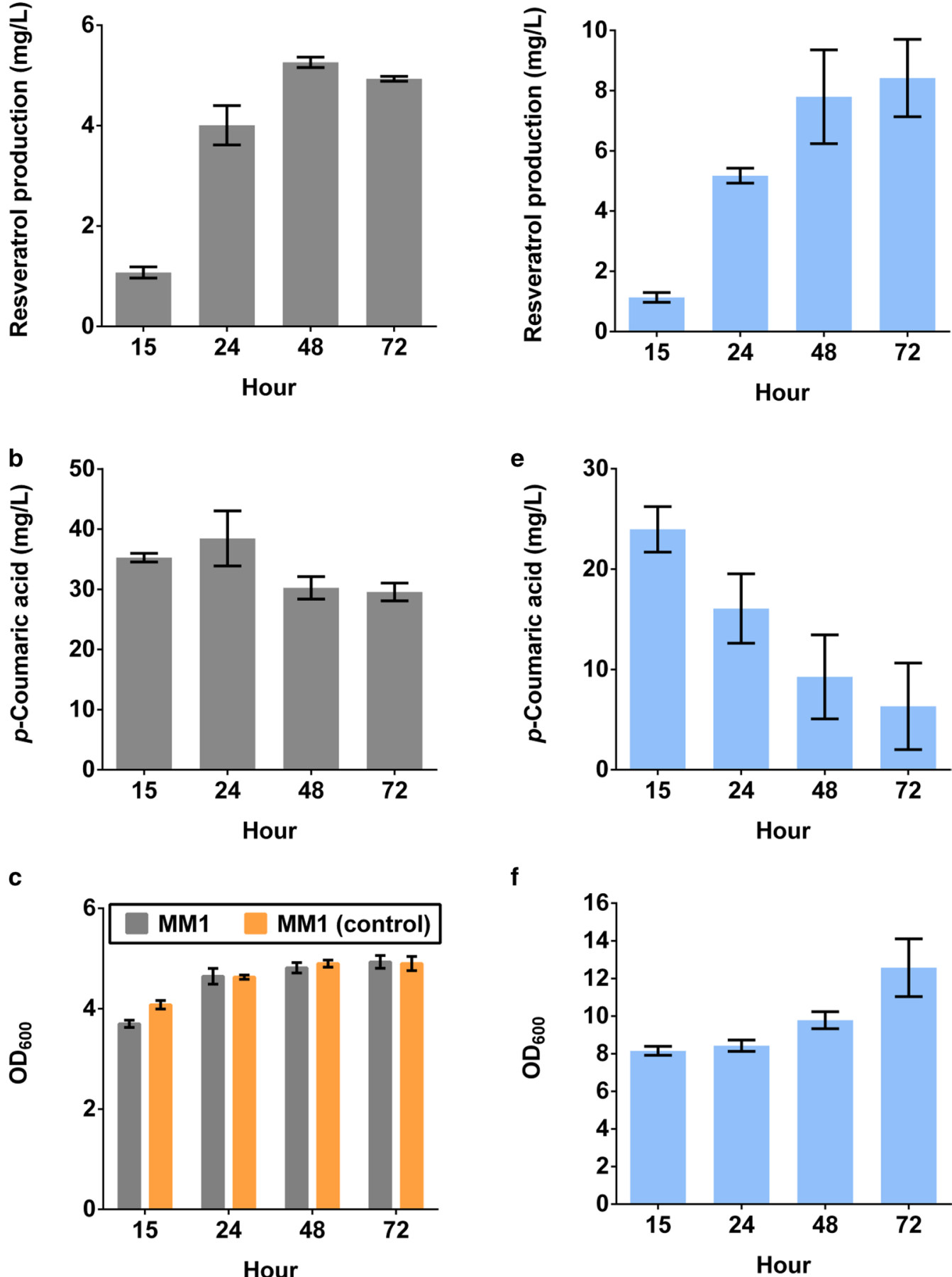

Fig. 2 De novo resveratrol production from glucose using the synthetic consortia. Comparisons of the consortia performance in a-c minimal media MM1, and $\mathbf{d}-\mathbf{f}$ complex media RM1. Time-course profiles of $\mathbf{a}$, $\mathbf{d}$ resveratrol production, $\mathbf{b}$, e accumulated $p$-coumaric acid as well as $\mathbf{c}, \mathbf{f}$ biomass formation. All media contain $20 \mathrm{~g} / \mathrm{L}$ glucose. $\mathbf{c}$ The growth status of a non-p-coumaric acid producer E. coli eBL0400DT-yeast sBY11 consortium was used as a control. The experiments were conducted with inoculation ratio of 1:1 and initial net cells density of $3 \times 10^{6}$ cells per $\mathrm{mL}$ of culture. Each data point and error bars represent means and standard deviations from biological triplicates, respectively 

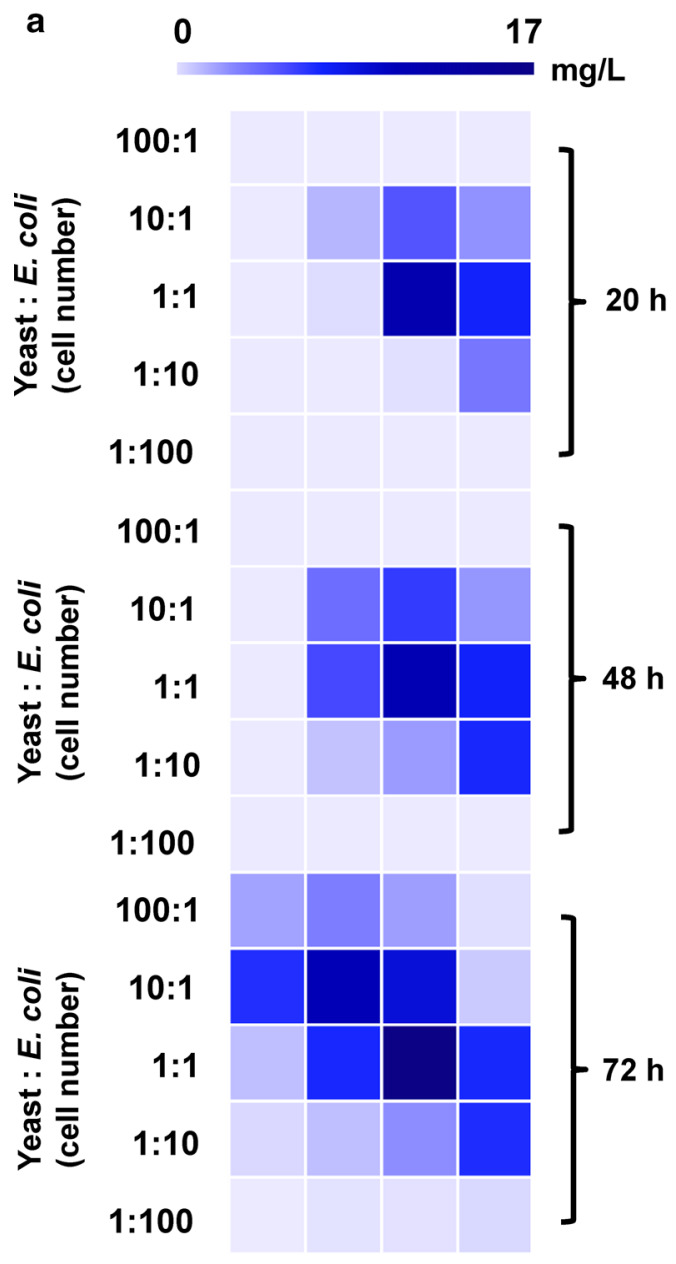

$\begin{array}{llll}25 & 30 & 33.5 & 37\end{array}$

Temperature $\left({ }^{\circ} \mathrm{C}\right)$

$\mathrm{h}$

$48 \mathrm{~h}$ b
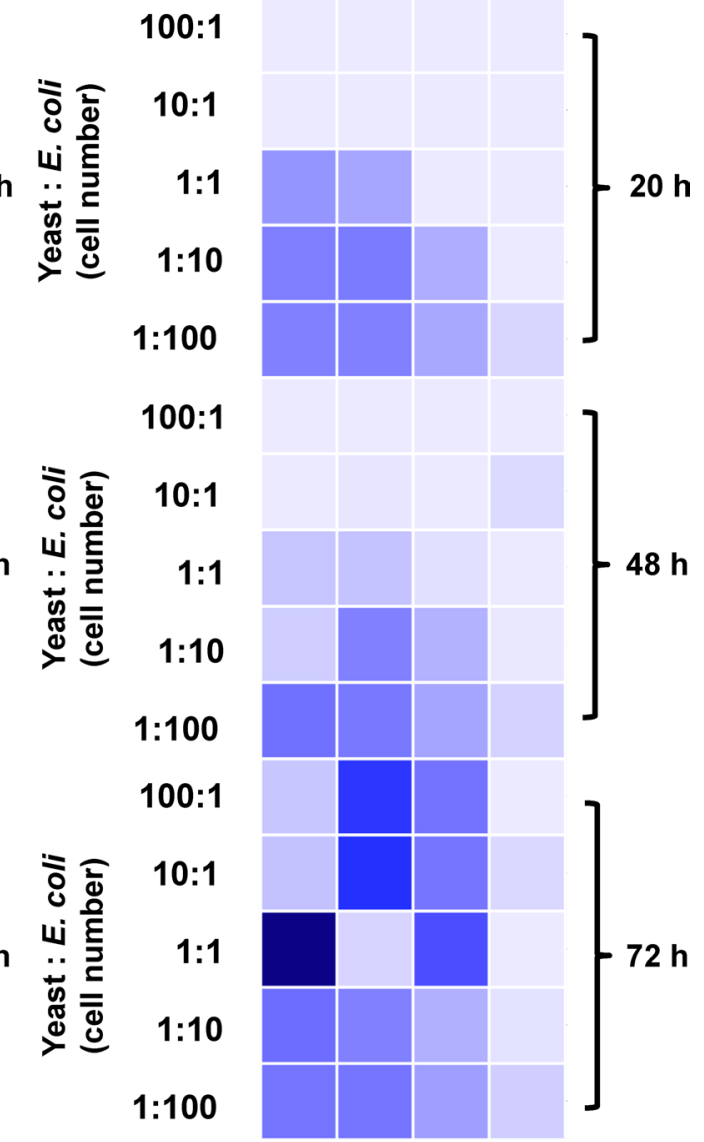

$\begin{array}{llll}25 & 30 & 33.5 & 37\end{array}$

Temperature $\left({ }^{\circ} \mathrm{C}\right)$

Fig. 3 Investigating the impact of fermentation conditions on resveratrol production with the use of test-tube screening. Heat maps for consortia performance of a resveratrol production and $\mathbf{b} p$-coumaric acid accumulation using co-cultures with altered inoculation cell number ratios, fermentation temperatures, and culture times. Experiments were conducted with a constant initial net cell density of $3 \times 10^{6}$ cells per mL. The color scale bar shown represents the concentration of indicated metabolites. Data points are mean values with $n=3$ biological replicates. Bar graphs containing this data with error bars can be found in the Additional file 1: Fig. S3

yeast strain was the rate limiting module for $p$-coumaric acid-to-resveratrol conversion, especially when operating the synthetic co-culture platform at a temperature range of $25-33.5{ }^{\circ} \mathrm{C}$. Among all the conditions, the inoculation ratio of 1:1 exhibited the highest averaged final resveratrol titer of $7.83 \mathrm{mg} / \mathrm{L}$ (Fig. 3a and Additional file 1: Fig. S3a-c), thus indicating that the 1:1 ratio was the optimal inoculation ratio for the synthetic consortia.

Using the information generated in this analysis, we were able to achieve a maximum resveratrol productivity of $0.57 \mathrm{mg} / \mathrm{L} / \mathrm{h}$ when fermentation was conducted with an inoculation ratio of $1: 1$ at $33.5{ }^{\circ} \mathrm{C}$ (Table 1 ).
Specifically, a maximum resveratrol titer of $16.6 \mathrm{mg} / \mathrm{L}$ was obtained along with $120.16 \mathrm{mg} / \mathrm{L} p$-coumaric acid accumulated at the end of the $72 \mathrm{~h}$ fermentation (Fig. 3a, $\mathrm{b}$ and Additional file 1: Fig. S3c, f). As a result, these conditions were used for a flask-scale up as discussed in the next section.

\section{Scale up resveratrol production using the synthetic consortia at a shake flask scale}

Process scale-up is an important aspect for industrial biofuel or biochemical production [32, 33]. Based on the optimal condition achieved at the test tube scale 
Table 1 Comparison of the effects of inoculation ratio and fermentation temperature on resveratrol productivity of the synthetic co-culture at a test tube scale with a constant initial net cell density $\left(3 \times 10^{6}\right.$ cells per mL of culture)

\begin{tabular}{|c|c|c|c|c|c|}
\hline \multirow{2}{*}{$\begin{array}{l}\text { Fermentation time } \\
\text { (hour) }\end{array}$} & \multirow{2}{*}{$\begin{array}{l}\text { Inoculation ratio } \\
\text { (Yeast: E. coli) }\end{array}$} & \multicolumn{4}{|c|}{ Resveratrol productivity (mg/L/h) } \\
\hline & & $25^{\circ} \mathrm{C}$ & $30^{\circ} \mathrm{C}$ & $33.5^{\circ} \mathrm{C}$ & $37^{\circ} \mathrm{C}$ \\
\hline \multirow[t]{5}{*}{20} & 100:1 & $0.00 \pm 0.00$ & $0.00 \pm 0.00$ & $0.00 \pm 0.00$ & $0.00 \pm 0.00$ \\
\hline & $10: 1$ & $0.00 \pm 0.00$ & $0.08 \pm 0.03$ & $0.24 \pm 0.08$ & $0.13 \pm 0.04$ \\
\hline & $1: 1$ & $0.00 \pm 0.00$ & $0.02 \pm 0.01$ & $0.57 \pm 0.04^{\mathrm{a}}$ & $0.35 \pm 0.02$ \\
\hline & $1: 10$ & $0.00 \pm 0.00$ & $0.00 \pm 0.00$ & $0.01 \pm 0.02$ & $0.18 \pm 0.02$ \\
\hline & 1:100 & $0.00 \pm 0.00$ & $0.00 \pm 0.00$ & $0.00 \pm 0.00$ & $0.00 \pm 0.00$ \\
\hline \multirow[t]{5}{*}{48} & 100:1 & $0.00 \pm 0.00$ & $0.00 \pm 0.00$ & $0.00 \pm 0.00$ & $0.00 \pm 0.00$ \\
\hline & $10: 1$ & $0.00 \pm 0.00$ & $0.08 \pm 0.01$ & $0.12 \pm 0.03$ & $0.05 \pm 0.01$ \\
\hline & $1: 1$ & $0.00 \pm 0.00$ & $0.11 \pm 0.01$ & $0.23 \pm 0.02$ & $0.15 \pm 0.01$ \\
\hline & $1: 10$ & $0.00 \pm 0.00$ & $0.02 \pm 0.00$ & $0.05 \pm 0.00$ & $0.14 \pm 0.02$ \\
\hline & 1:100 & $0.00 \pm 0.00$ & $0.00 \pm 0.00$ & $0.00 \pm 0.00$ & $0.00 \pm 0.00$ \\
\hline \multirow[t]{5}{*}{72} & 100:1 & $0.03 \pm 0.01$ & $0.05 \pm 0.01$ & $0.03 \pm 0.01$ & $0.00 \pm 0.00$ \\
\hline & 10:1 & $0.09 \pm 0.02$ & $0.15 \pm 0.05$ & $0.12 \pm 0.05$ & $0.01 \pm 0.01$ \\
\hline & $1: 1$ & $0.02 \pm 0.00$ & $0.09 \pm 0.02$ & $0.23 \pm 0.02$ & $0.09 \pm 0.01$ \\
\hline & $1: 10$ & $0.01 \pm 0.01$ & $0.02 \pm 0.01$ & $0.04 \pm 0.02$ & $0.09 \pm 0.00$ \\
\hline & 1:100 & $0.00 \pm 0.00$ & $0.00 \pm 0.00$ & $0.00 \pm 0.00$ & $0.01 \pm 0.00$ \\
\hline
\end{tabular}

a Denotes that the condition with a maximum productivity of resveratrol

(16.6 $\mathrm{mg} / \mathrm{L}$ at $72-\mathrm{h}$ timepoint using a $1: 1$ inoculation ratio with $33.5{ }^{\circ} \mathrm{C}$ temperature), we sought to evaluate the scalability of this synthetic co-culture at a shake flask scale with an extended fermentation period (96 h). To this end, we measured resveratrol production, accumulated $p$-coumaric acid level, and co-culture growth profile across more timepoints (Fig. 4 and Table 2). In this condition, we found a 1.68-fold improvement at flask scale resulting in a maximum resveratrol productivity of $0.96 \mathrm{mg} / \mathrm{L} / \mathrm{h}$ (Fig. $4 \mathrm{a}$ and Table 2). Additionally, a maximum resveratrol titer of $28.5 \mathrm{mg} / \mathrm{L}$ was achieved at the flask scale (Fig. 4a), which was 1.72-fold higher than that at the test tube scale. This result implied that the synthetic consortium was more metabolically active for resveratrol production at the flask scale than that at the test tube scale, possibly due to better aeration, mass transfer and agitation provided in a shake flask [34, 35]. However, resveratrol gradually decreased after $48 \mathrm{~h}$ accompanied by an increase in $p$-coumaric acid accumulation (Fig. 4a, b). Furthermore, a relatively lower biomass formation was seen in this engineered $p$-coumaric acid E. coli eBL0430T-yeast sBY11 co-culture compared to a control co-culture containing non- $p$-coumaric acid producer E. coli eBL0400DT and engineered S. cerevisiae sBY11 strains (Fig. 4c). These results highlight a potential challenge with yeast as a production host in that resveratrol exhibits better antifungal (with minimum inhibitory concentrations (MICs) of 10-20 $\mu \mathrm{g} / \mathrm{mL}$ for S. cerevisiae) than antibacterial activity (with MICs of $57-1000 \mu \mathrm{g} / \mathrm{mL}$ for $E$. coli depending on species) [36, 37]. As a result, we tested higher inoculum sizes to prevent growth being influenced by this molecule.

\section{Increased net inoculum size of the co-culture improves resveratrol production}

Previous studies have shown that utilizing high cell density microbial bioprocesses can increase volumetric productivity and alleviate the impact of toxic growth inhibitors [32, 38, 39]. Similarly, increasing co-culture inoculum size of $S$. cerevisiae and E. coli led to an improvement in naringenin production [31]. Therefore, we chose to investigate whether maintaining the same optimal inoculation ratio describe above (1:1) with a tenfold higher net cells density (namely from $3 \times 10^{6}$ to $3 \times 10^{7}$ cells per $\mathrm{mL}$ of culture) could enhance resveratrol production. To comprehensively investigate the impact of increasing inoculum size on consortia performance, we finally performed the fermentations at a range of temperature $25-37{ }^{\circ} \mathrm{C}$ in a test tube scale and measured resveratrol production as well as accumulated $p$-coumaric acid level (Fig. 5).

Interestingly, at these higher inoculum sizes, an increased maximum resveratrol productivity of $0.63 \mathrm{mg} / \mathrm{L} / \mathrm{h}$ was achieved at $30{ }^{\circ} \mathrm{C}$ (Table 3 ) compared to the lower-density test tube experiment where the maximum was at $33.5{ }^{\circ} \mathrm{C}$ (Table 1). Furthermore, the co-culture seeded at a higher initial net cells density yielded a maximum resveratrol titer of $36.0 \mathrm{mg} / \mathrm{L}$ at $30{ }^{\circ} \mathrm{C}$ (Fig. 5a), a value that was nearly 5.35 -fold higher than the lower cell density condition $(6.74 \mathrm{mg} / \mathrm{L}$ ) (Fig. 3a and Additional 

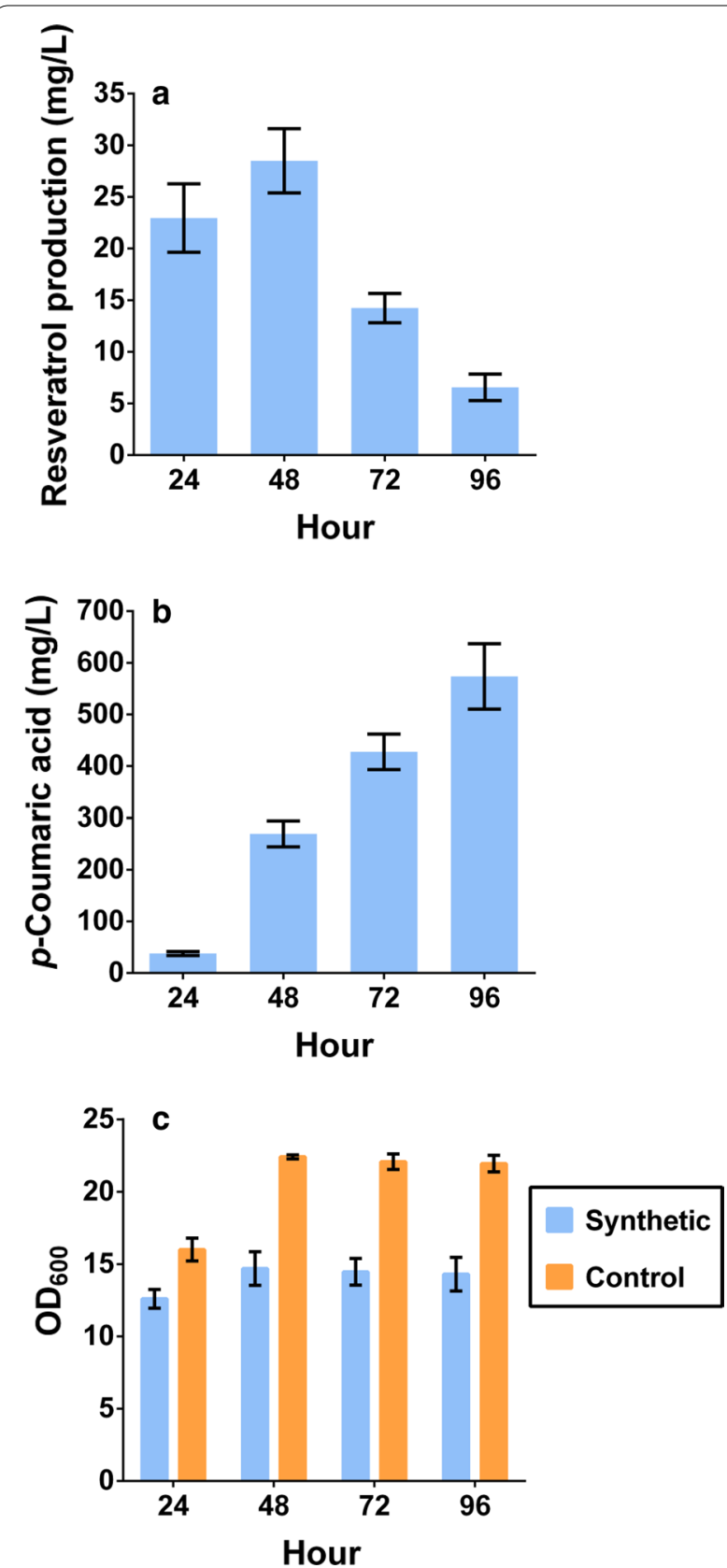

Fig. 4 Evaluation of the synthetic co-culture capacity for resveratrol production in flasks. Comparisons of a resveratrol production, $\mathbf{b}$ accumulated $p$-coumaric acid and $\mathbf{c}$ growth profile (labeled as Synthetic) for the designed E. coli eBL0430T-yeast sBY11 co-culture at $33.5^{\circ} \mathrm{C}$ are shown. $\mathbf{c}$ The growth dynamics for a non-p-coumaric acid producer E. coli eBL0400DT-yeast SBY11 consortium (labeled as Control) were similarly cultivated at $33.5^{\circ} \mathrm{C}$ to be used as a control. The experiments were conducted with constant inoculation ratio of 1:1 and initial net cells density of $3 \times 10^{6}$ cells per $\mathrm{mL}$ of culture. Each data point and error bar represent means and standard deviations from biological triplicates, respectively
Table 2 Resveratrol productivity of the synthetic co-culture at a shake flask scale with inoculation ratio (1:1), initial net cells density $\left(3 \times 10^{6}\right.$ cells per $\mathrm{mL}$ of culture), and $33.5^{\circ} \mathrm{C}$ temperature

\begin{tabular}{ll}
\hline Fermentation time & Resveratrol productivity $(\mathbf{m g} / \mathbf{L} / \mathbf{h})$ \\
\hline $24 \mathrm{~h}$ & $0.96 \pm 0.14^{\mathrm{a}}$ \\
$48 \mathrm{~h}$ & $0.59 \pm 0.07$ \\
$72 \mathrm{~h}$ & $0.20 \pm 0.02$ \\
$96 \mathrm{~h}$ & $0.07 \pm 0.01$
\end{tabular}

a Denotes that the condition with a maximum productivity of resveratrol
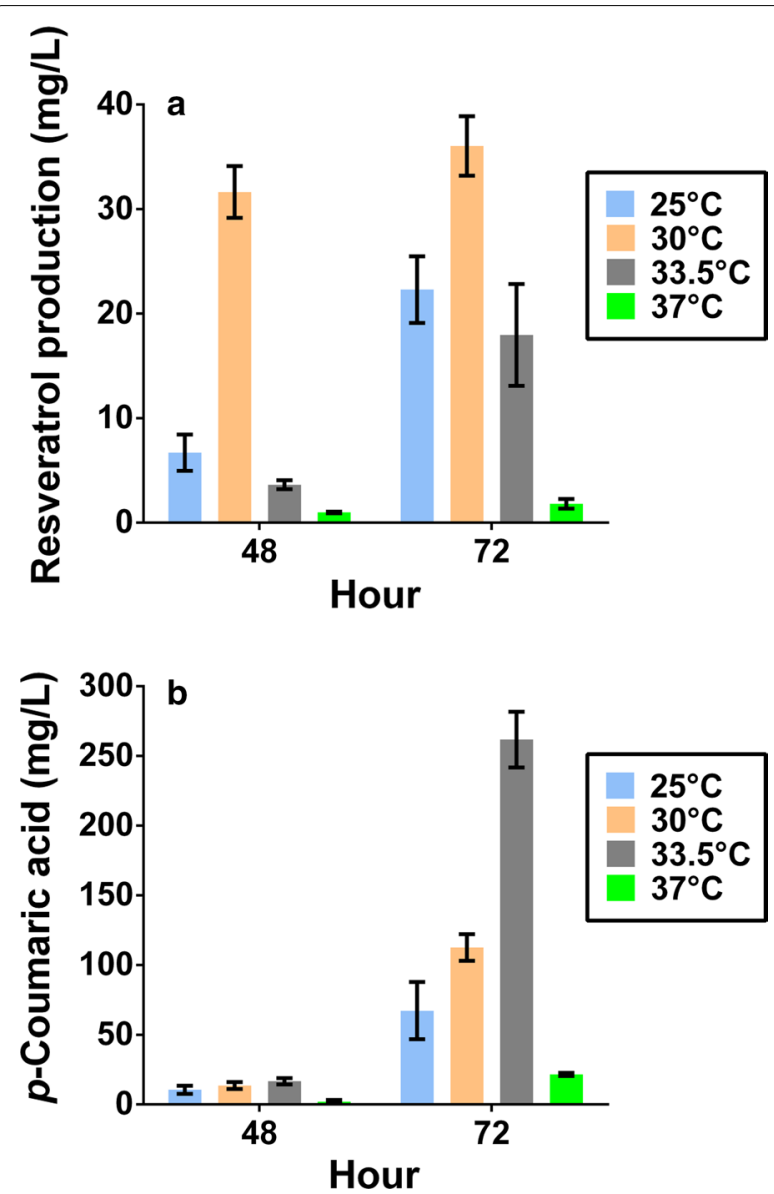

Fig. 5 Investigating the impact of increasing initial net inoculation cell density on co-culture performance. Comparisons of a resveratrol production and $\mathbf{b}$ accumulated $p$-coumaric acid for the synthetic co-culture was conducted at various temperatures $\left(25-37^{\circ} \mathrm{C}\right)$. The experiments were conducted with a constant inoculation ratio of 1:1 while changing the initial net cells density to $3 \times 10^{7}$ cells per $\mathrm{mL}$ of culture. Each data point and error bar represent means and standard deviations from biological triplicates, respectively 
Table 3 Comparison of the effects of fermentation temperature on resveratrol productivity of the synthetic co-culture at a test tube scale with a constant inoculation ratio (1:1) and initial net cell density $\left(3 \times 10^{7}\right.$ cells per $\mathrm{mL}$ of culture)

\begin{tabular}{lllll}
\hline $\begin{array}{l}\text { Fermentation } \\
\text { time }\end{array}$ & \multicolumn{4}{l}{ Resveratrol productivity $(\mathbf{m g} / \mathbf{L} / \mathbf{h})$} \\
\cline { 2 - 5 } & $\mathbf{2 5 ^ { \circ } \mathbf { C }}$ & $\mathbf{3 0}{ }^{\circ} \mathbf{C}$ & $\mathbf{3 3 . 5}{ }^{\circ} \mathbf{C}$ & $\mathbf{3 7}{ }^{\circ} \mathbf{C}$ \\
\hline $48 \mathrm{~h}$ & $0.14 \pm 0.04$ & $0.63 \pm 0.06^{\mathrm{a}}$ & $0.08 \pm 0.01$ & $0.02 \pm 0.00$ \\
$72 \mathrm{~h}$ & $0.31 \pm 0.04$ & $0.50 \pm 0.04$ & $0.25 \pm 0.07$ & $0.03 \pm 0.01$ \\
\hline
\end{tabular}

${ }^{a}$ Denotes that the condition with a maximum productivity of resveratrol

file 1: Fig. S3c). It should be noted that only $18.0 \mathrm{mg} / \mathrm{L}$ resveratrol was produced at $33.5^{\circ} \mathrm{C}$, the optimal temperature identified at a lower cell density seeding (Fig. 3a). Moreover, less accumulation of $p$-coumaric acid was observed $(112.5 \mathrm{mg} / \mathrm{L})$ when the fermentation was performed at $30^{\circ} \mathrm{C}$ for $72 \mathrm{~h}$ when compared with the $33.5^{\circ} \mathrm{C}$ condition (261.8 mg/L) (Fig. 5b). Additionally, the averaged resveratrol titer of the conditions conducted at the lower temperature range $25-33.5^{\circ} \mathrm{C}$ for $72 \mathrm{~h}(25.4 \mathrm{mg} / \mathrm{L})$ (Fig. 5a) was nearly 3.1 -fold higher than the corresponding lower initial cell density conditions $(8.2 \mathrm{mg} / \mathrm{L}$ ) (Additional file 1: Fig. S3c). These results seem to indicate that the downstream yeast module was more metabolically active for conversion of $p$-coumaric acid into the end product at $30^{\circ} \mathrm{C}$ than that at $33.5^{\circ} \mathrm{C}$ when using high cell density fermentation strategy. As a result, this discrepancy in temperature optimum likely results from changes in the end-point population dynamics and metabolic potential caused by differences in initial starting density.

\section{Conclusions}

In this study, we first establish a novel E. coli-S. cerevisiae consortium platform for de novo resveratrol biosynthesis from glucose via modular co-culture engineering. Through optimization of fermentation parameters, including altering inoculation ratios of co-culture, cultivation temperatures and times, we found that the downstream yeast module is a rate limiting node for $p$-coumaric acid-to-resveratrol conversion. This unique consortium enables resveratrol production with a high titer of $28.5 \mathrm{mg} / \mathrm{L}$ at a shake flask scale that can be improved to $36 \mathrm{mg} / \mathrm{L}$ resveratrol in a test tube when using higher cell density inoculations. While this work was conducted at a small scale, they nevertheless point toward advances in the use of co-cultures and exceed the value of another E. coli-S. cerevisiae co-culture for a similar polyketide, naringenin (obtaining $21 \mathrm{mg} / \mathrm{L}$ ) [31]. These findings advance complex natural product biosynthesis with the use of bacterium-yeast co-culture approach. Several genetic strategies could further enhance resveratrol production using our E. coli-S. cerevisiae co-culture scenario, including (i) driving more metabolic flux from precursor $p$-coumaric acid toward resveratrol biosynthesis through multicopy integration of resveratrol biosynthetic genes $4 C L$ and STS into longterminal repeat (LTR)-retrotransposons of $S$. cerevisiae such as Ty1 or Ty4 elements [10, 40], (ii) scaling up the fermentation in a fed-batch controlled mode via monitoring co-culture growth status and constantly feeding low level of glucose to avoid overflow metabolism (i.e. formation of side metabolites such as acetate for E. coli and ethanol for S. cerevisiae), and (iii) executing more sophisticated genetic manipulations for creating a mutualistic consortium to further reduce metabolite inhibitions on consortia growth and thus improve the final yield (i.e. S. cerevisiae can only utilize acetate excreted by E. coli when using xylose as a carbon source without producing ethanol [41], or rewiring metabolism of $S$. cerevisiae to render a Crabtree-negative yeast [42]). Finally, on a molecular side, addressing limited bioavailability of this molecule by attaching sugar moieties to resveratrol can improve stability and solubility in aqueous solutions [43, 44]. Nevertheless, these results demonstrate the viability of a co-culture approach for production of resveratrol.

\section{Materials and methods \\ Strains, media and plasmid or integrative expression cassette construction}

All strains and plasmids used in this study are listed in Table 4. Sequences of primers synthesized by Integrated DNA Technologies (Coralville, IA) and codon-optimized DNA fragments used in this work are listed in Additional file 1: Tables S1 and S2, respectively. All Gibson-assembled DNA [45] were electroporated (2 mm Electroporation Cuvettes, Bioexpress) into E. coli competent cells with a BioRad Genepulser Xcell at $2.5 \mathrm{kV}$. E. coli NEB10 $\beta$ was used for gene cloning or propagation of all expression vectors. It was cultivated in Luria-Bertani (LB) medium ( $1 \%$ tryptone, $0.5 \%$ yeast extract and $1 \% \mathrm{NaCl}$ ) supplemented with appropriate antibiotics $(50 \mu \mathrm{g} / \mathrm{mL}$ kanamycin or $50 \mu \mathrm{g} / \mathrm{mL}$ spectinomycin (Sigma)) with $225 \mathrm{rpm}$ orbital shaking at $37^{\circ} \mathrm{C}$. The Frozen EZ Yeast Transformation II Kit (Zymo Research) was used to transform an integrative expression cassette into the yeast, and the resulting yeast transformants were selected on yeast synthetic complete (YSC) media with the appropriate dropouts for auxotrophic selection. RM1 (1X CSM-URA-LEU (MP Biomedicals), 1X Yeast Nitrogen Base (BD Difco) at $5 \mathrm{~g} / \mathrm{L}$ of ammonium sulfate, $1 \%$ tryptone, $0.5 \%$ yeast extract, $1 \% \mathrm{NaCl}, 20 \mathrm{~g} / \mathrm{L}$ D-glucose, $50 \mu \mathrm{g} / \mathrm{mL}$ kanamycin and $50 \mu \mathrm{g} / \mathrm{mL}$ spectinomycin) and MM1 (1X CSM-URALEU (MP Biomedicals), 1X Yeast Nitrogen Base (BD Difco) at $5 \mathrm{~g} / \mathrm{L}$ of ammonium sulfate, $1 \mathrm{X}$ M9 minimal 
Table 4 List of strains and plasmids used in this study

\begin{tabular}{|c|c|c|}
\hline Strain/plasmid & Description & Source \\
\hline \multicolumn{3}{|l|}{ E. coli strain } \\
\hline NEB10 $\beta$ & $\begin{array}{l}\Delta \text { (ara-leu) } 7697 \text { araD139 fhuA } \triangle \text { lacX74 galK16 galE15 e14- } 480 d \text { lacZ } \triangle M 15 \text { recA1 relA1 endA1 nup } \\
\left.\text { rpsL (Str }{ }^{R}\right) \text { rph spoT1 } \triangle(\text { mrr-hsdRMS-mcrBC) }\end{array}$ & New England Biolabs \\
\hline BL21(DE3) & $\begin{array}{l}\text { E. coli str. B F }{ }^{-} \text {ompT gal dem lon hsdS }{ }_{B}\left(r_{B}^{-} m_{B}^{-}\right) \lambda\left(D E 3 \text { [lacl lacUV5-T7p07 ind1 sam7 nin5]) }\left[\mathrm{malB}^{+}\right]_{\mathrm{K}-}\right. \\
\end{array}$ & New England Biolabs \\
\hline eBL04 & [BL21(DE3)] $\Delta$ tyrR pET28-pYIBN-aroG G(br)-B30rbs-tyra (fbr)-tRRNC; Kan ${ }^{R}$ & [28] \\
\hline eBL0400DT & [eBL04] pCDFDuet-1; Kan $;$ Spc $^{R}$ & [28] \\
\hline eBL0430T & [eBL04] pCDF-pLPP-B30rbs-TcXAL-T7t; Kan ${ }^{R} ;$ Spc $^{R}$ & This study \\
\hline eBL0432T & [eBL04] pCDF-pLPP-B32rbs-TCXAL-T7t; Kan ${ }^{R} ; S p c^{R}$ & This study \\
\hline \multicolumn{3}{|l|}{ S. cerevisiae strain } \\
\hline BY4741 & MATa SUC2 gal2 mal2 mel flo 1 flo8-1 hap 1 ho bio 1 bio6 his $3 \Delta 1$ leu2 $\Delta 0$ met15 $\Delta 0$ ura3 $\Delta 0$ & ATCC \\
\hline sBY10 & $\begin{array}{l}\text { [BY4741] pTEF1-At4CL-tADH1-pPGK1-VVSTS-tCYC1 (integration with Kluyveromyces lactis LEU2 } \\
\text { marker) }\end{array}$ & This study \\
\hline sBY11 & 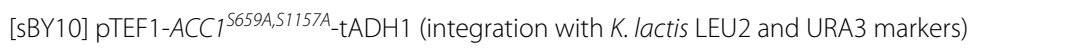 & This study \\
\hline \multicolumn{3}{|l|}{ Plasmids } \\
\hline pCDF-Duet-1 & For construction $p$-coumaric acid producing plasmids & [28] \\
\hline pETM-TAL-4CL & For amplification of TCXAL gene & [30] \\
\hline pCfB1020 & For construction resveratrol expression cassette & [10] \\
\hline pCfB1175 & For construction $\mathrm{ACC} 1$ mutant expression cassette & {$[10]$} \\
\hline pCDF-pLPP-B30rbs-TCXAL-T7t & For $p$-coumaric acid production testing & This study \\
\hline pCDF-pLPP-B32rbs-TcXAL-T7t & For $p$-coumaric acid production testing & This study \\
\hline
\end{tabular}

salts (Sigma), $2 \mathrm{mM} \mathrm{MgSO}, 0.1 \mathrm{mM} \mathrm{CaCl} 2,20 \mathrm{~g} / \mathrm{L}$ D-glucose, $50 \mu \mathrm{g} / \mathrm{mL}$ kanamycin and $50 \mu \mathrm{g} / \mathrm{mL}$ spectinomycin) were used for medium optimization studies.

For construction of pCDF-pLPP-B30rbs-TcXAL-T7t, Gibson assembly method was employed to combine an amplicon containing TAL gene amplified from pETMTAL-4CL [30] with primers P80 as well as P81, primer P67, and the PCR product amplified from pCDF-Duet-1 empty vector [28] with primers 82 and 83 . To construct pCDF-pLPP-B32rbs-TcXAL-T7t, the DNA fragment PCR-amplified from pETM-TAL-4CL [30] with primers P84 as well as P81, was Gibson assembled with the primer $\mathrm{P} 68$ and the amplicon amplified from pCDFDuet-1 empty vector [28] with primers 82 as well as 83 . To generate a yeast integrative expression cassette carrying 4CL and STS genes, primers P85 and P86 were used for amplifying a linear DNA fragment from pCfB1020 [10]. To yield an integrative cassette containing posttranslational deregulated $A C C 1^{S 659 A, S 1157 A}$ gene, primers P89 and P90 were used for amplifying a linear DNA fragment from pCfB1175 [10].

\section{Upstream E. coli module construction and $p$-coumaric acid production}

To construct the upstream module of the design co-culture, the $p$-coumaric acid producing plasmids pCDF-pLPPB30rbs-TcXAL-T7t and pCDF-pLPP-B32rbs-TcXAL-T7t were individually transformed into eBL04 [28] leading to eBL0430T and eBL0432T strains, respectively. The resulting strains were able to constitutively overexpress $T$. cutaneum TAL enzyme. For $p$-coumaric acid production, starter culture of $E$. coli was grown in $2 \mathrm{~mL} \mathrm{LB}$ medium containing $50 \mu \mathrm{g} / \mathrm{mL}$ kanamycin and $50 \mu \mathrm{g} / \mathrm{mL}$ spectinomycin with $225 \mathrm{rpm}$ orbital shaking at $30{ }^{\circ} \mathrm{C}$ overnight. Then seed culture was inoculated into $3 \mathrm{~mL} \mathrm{LB}$ medium supplemented with antibiotics with an initial $\mathrm{OD}_{600}$ of 0.05 and incubated at $30{ }^{\circ} \mathrm{C}$ for $18 \mathrm{~h}$. After fermentation, suspension culture was mixed with equal volume of absolute ethanol and centrifuged at $16,000 \mathrm{~g}$ for $2 \mathrm{~min}$. The supernatant fraction was collected for measurement of $p$-coumaric acid production using HPLC. The cell growth was measured by Ultrospec 2100 Pro UV/Visible Spectrophotometer observing optical density at $600 \mathrm{~nm}$.

\section{Downstream yeast module construction and resveratrol production}

To construct the downstream module of the design coculture, we first integrated the constitutive expression cassette containing heterologous $4 C L$ and STS genes into $S$. cerevisiae $\mathrm{MCH} 2$ locus located at chromosome XI [10]. The resulting transformants were selected on YSC dropout media (CSM-LEU) for auxotrophic selection and verified by colony PCR with primers P87 and P88. The resulting yeast strain, designated as sBY10, was 
subsequently transformed with an integrated expression cassette harboring S. cerevisiae ACC1 $1^{\text {S659A,S1157A }}$ gene and homology arms targeting to an insertion site located between NCA3 and ASF1 loci at S. cerevisiae chromosome $X$ [10]. The resulting transformants were selected on YSC dropout media (CSM-URA-LEU) and verified by colony PCR with primers P91 and P92, leading to a yeast strain sBY11. Prior to all fermentation tests, the starter E. coli and yeast cultures were inoculated from glycerol stocks into $3 \mathrm{~mL} \mathrm{LB}$ (supplemented with $50 \mu \mathrm{g} / \mathrm{mL}$ kanamycin and $50 \mu \mathrm{g} / \mathrm{mL}$ spectinomycin) and YSC dropout media (CSM-URA-LEU), respectively. All seeding cultures were incubated at $30{ }^{\circ} \mathrm{C}$.

For optimization of fermentation medium, E. coli eBL0430T was co-cultured with S. cerevisiae SBY11 and its capacity of resveratrol production was evaluated using RM1 or MM1 medium. The fermentation was performed with seeding the same initial cell density of $1.5 \times 10^{6}$ cells $/ \mathrm{mL}$ for each strain in test tubes containing $3 \mathrm{~mL}$ RM1 or MM1 medium. The cultures were afterwards incubated at $33.5^{\circ} \mathrm{C}$. A non-p-coumaric acid producer $E$. coli eBL0400DT [28] cocultured with yeast sBY11 strain was used as a control consortium (Fig. $2 \mathrm{c}$ and Additional file 1: Fig.S2). To investigate the impacts of inoculation ratios, fermentation temperatures and times on resveratrol production, various yeast: E. coli cell ratios (100:1, 10:1, 1:1, 1:10 and 1:100 with a constant initial net cells density of $3 \times 10^{6}$ cells per $\mathrm{mL}$ of culture) as well as temperatures $\left(25,30,33.5\right.$ and $\left.37^{\circ} \mathrm{C}\right)$ were adopted for production testing in test tubes containing $3 \mathrm{~mL}$ RM1 media. For evaluating the co-culture performance at a shake flask scale, fermentations were carried out at $33.5{ }^{\circ} \mathrm{C}$ using $25 \mathrm{~mL}$ RM1 media in $125 \mathrm{~mL}$-flasks. All conditions were conducted at an inoculation ratio of $1: 1$ with a constant initial net cells density of $3 \times 10^{6}$ cells per $\mathrm{mL}$ of culture. To investigate the effect of increasing initial net cells density on the consortia's capacity for $p$-coumaricto-resveratrol conversion, initial net coculture inoculum was increased from original $3 \times 10^{6}$ to $3 \times 10^{7}$ cells per $\mathrm{mL}$ of culture, and fermentations were performed at various temperatures $\left(25,30,33.5\right.$ and $\left.37^{\circ} \mathrm{C}\right)$ while keeping the inoculation ratio constant at 1:1.

All timepoint samples were mixed with equal volume of absolute ethanol and centrifuged at 16,000 $\mathrm{g}$ for $2 \mathrm{~min}$. The supernatants were used to analyze resveratrol and $p$-coumaric acid by HPLC.

\section{HPLC analysis}

Samples from fermentations were filtered with $0.2-\mu \mathrm{m}$ nylon syringe filters (Wheaton Science) prior to running HPLC. HPLC confirmation of resveratrol or $p$-coumaric acid production was performed using a Dionex UltiMate 3000 (Thermo Fisher Scientific) equipped with an Agilent
Eclipse Plus C18 column $(3.0 \times 150 \mathrm{~mm}, 3.5 \mu \mathrm{m})$ with detection wavelength at $304 \mathrm{~nm}$. Column oven was held at $25{ }^{\circ} \mathrm{C}$ with $1 \%$ acetic acid in water or acetonitrile as the mobile phase over the course of the 20-min sequence under the following conditions: $5 \%$ to $15 \%$ organic (vol/ vol) for $5 \mathrm{~min}, 15 \%$ to $100 \%$ organic (vol/vol) for $8 \mathrm{~min}$, $100 \%$ organic (vol/vol) for $2 \mathrm{~min}, 100 \%$ to $5 \%$ organic for $2 \mathrm{~min}$ followed by $5 \%$ organic for $3 \mathrm{~min}$. The constant flow rate was set at $0.8 \mathrm{~mL}$ min-1. A standard curve was prepared using $\geq 99 \%$ purity resveratrol or $\geq 98.0 \%$ purity $p$-coumaric acid from Sigma-Aldrich.

\section{Supplementary information}

Supplementary information accompanies this paper at https://doi. org/10.1186/s12934-020-01401-5.

Additional file 1.

Acknowledgements

This work was supported by the Camille and Henry Dreyfus Foundation. We thank Dr. Jens Nielsen for providing the pCfB1020 and pCfB1175 plasmids. We also thank Dr. Shenghu Zhou for providing the pETM-TAL-4CL.

\section{Authors' contributions}

HSA and SFY conceived the project, analyzed results, and wrote the manuscript. SFY designed a consortium E. coli-yeast for de novo resveratrol biosynthesis. SFY conducted resveratrol fermentation with contributions from $X Y$ and TGJ. All authors read and approved the final manuscript.

Availability of data and materials

All data generated or analyzed in this study are included in the manuscript and its additional file.

Ethics approval and consent to participate

Not applicable.

Consent for publication

Not applicable.

\section{Competing interests}

The authors declare that they have no competing interests.

\section{Author details}

${ }^{1}$ Institute for Cellular and Molecular Biology, The University of Texas at Austin, Austin, TX, USA. ${ }^{2}$ Department of Chemistry, University of Washington,

Box 351700, Seattle, WA, USA. ${ }^{3}$ McKetta Department of Chemical Engineering, The University of Texas at Austin, 200 E Dean Keeton St. Stop C0400, Austin, TX 78712, USA.

Received: 25 May 2020 Accepted: 7 July 2020

Published online: 14 July 2020

\section{References}

1. Rodriguez-Casado A. The health potential of fruits and vegetables phytochemicals: notable examples. Crit Rev Food Sci Nutr. 2016;56:1097-107.

2. Gambini J, Inglés M, Olaso G, Lopez-Grueso R, Bonet-Costa V, GimenoMallench L, Mas-Bargues C, Abdelaziz KM, Gomez-Cabrera MC, Vina $J$, Borras C. Properties of resveratrol: in vitro and in vivo studies about metabolism, bioavailability, and biological effects in animal models and humans. Oxid Med Cell Longevity. 2015;2015:837042.

3. Rotches-Ribalta M, Andres-Lacueva C, Estruch R, Escribano E, Urpi-Sarda $M$. Pharmacokinetics of resveratrol metabolic profile in healthy humans 
after moderate consumption of red wine and grape extract tablets. Pharmacol Res. 2012;66:375-82.

4. Yuan S-F, Alper HS. Metabolic engineering of microbial cell factories for production of nutraceuticals. Microb Cell Fact. 2019;18:46.

5. Global Resveratrol Market Research Report 2020. Industry Research. 2020:https://www.industryresearch.co/global-resveratrol-market-15064 120.

6. Farina A, Ferranti C, Marra C. An improved synthesis of resveratrol. Nat Prod Res. 2006:20:247-52.

7. Wang D-G, Liu W-Y, Chen G-T. A simple method for the isolation and purification of resveratrol from Polygonum cuspidatum. J Pharma Anal. 2013;3:241-7.

8. Tian B, Liu J. Resveratrol: a review of plant sources, synthesis, stability, modification and food application. J Sci Food Agric. 2020;100:1392-404.

9. Kallscheuer N, Vogt M, Stenzel A, Gätgens J, Bott M, Marienhagen J. Construction of a Corynebacterium glutamicum platform strain for the production of stilbenes and (2S)-flavanones. Metab Eng. 2016;38:47-55.

10. Li M, Kildegaard KR, Chen Y, Rodriguez A, Borodina I, Nielsen J. De novo production of resveratrol from glucose or ethanol by engineered Saccharomyces cerevisiae. Metab Eng. 2015;32:1-11.

11. Li M, Schneider K, Kristensen M, Borodina I, Nielsen J. Engineering yeast for high-level production of stilbenoid antioxidants. Sci Rep. 2016;6:36827.

12. Palmer CM, Miller KK, Nguyen A, Alper HS. Engineering 4-coumaroylCoA derived polyketide production in Yarrowia lipolytica through a B-oxidation mediated strategy. Metab Eng. 2020;57:174-81.

13. Lim CG, Fowler ZL, Hueller T, Schaffer S, Koffas MAG. High-yield resveratrol production in engineered Escherichia coli. Appl Environ Microbiol. 2011;77:3451

14. Hong J, Im D-K, Oh M-K. Investigating E. coli coculture for resveratrol production with $13 \mathrm{C}$ metabolic flux analysis. J Agri Food Chem. 2020;68:3466-73.

15. Wang J, Yang Y, Yan Y. Bioproduction of Resveratrol. In: Schwab W, Lange BM, Wüst M, editors. Biotechnology of natural products. Cham: Springer International Publishing; 2018. p. 61-79.

16. Barros J, Dixon RA. Plant phenylalanine/tyrosine ammonia-lyases. Trends Plant Sci. 2020;25:66-79.

17. Thapa BS, Pandey PR, Park IY, Sohng KJ. Biotechnological advances in resveratrol production and its chemical diversity. Molecules. 2019;24:2571.

18. Park SR, Yoon JA, Paik JH, Park JW, Jung WS, Ban Y-H, Kim EJ, Yoo YJ, Han $A R$, Yoon YJ. Engineering of plant-specific phenylpropanoids biosynthesis in Streptomyces venezuelae. J Biotechnol. 2009:141:181-8.

19. Sydor T, Schaffer S, Boles E. Considerable increase in resveratrol production by recombinant industrial yeast strains with use of rich medium. Appl Environ Microbiol. 2010;76:3361.

20. Sun L, Alper HS. Non-conventional hosts for the production of fuels and chemicals. Curr Opin Chem Biol. 2020;59:15-22.

21. Liu X, Li X-B, Jiang J, Liu Z-N, Qiao B, Li F-F, Cheng J-S, Sun X, Yuan Y-J, Qiao J, Zhao G-R. Convergent engineering of syntrophic Escherichia coli coculture for efficient production of glycosides. Metab Eng. 2018;47:243-53.

22. Roell GW, Zha J, Carr RR, Koffas MA, Fong SS, Tang YJ. Engineering microbial consortia by division of labor. Microb Cell Fact. 2019;18:35.

23. McCarty NS, Ledesma-Amaro R. Synthetic biology tools to engineer microbial communities for biotechnology. Trends Biotechnol. 2019:37:181-97.

24. Chen Z, Sun X, Li Y, Yan Y, Yuan Q. Metabolic engineering of Escherichia coli for microbial synthesis of monolignols. Metab Eng. 2017;39:102-9.

25. Zhang H, Wang X. Modular co-culture engineering, a new approach for metabolic engineering. Metab Eng. 2016;37:114-21.

26. Jones $J A$, Wang $X$. Use of bacterial co-cultures for the efficient production of chemicals. Curr Opin Biotechnol. 2018;53:33-8.

27. Camacho-Zaragoza JM, Hernández-Chávez G, Moreno-Avitia F, RamírezIñiguez R, Martínez A, Bolívar F, Gosset G. Engineering of a microbial coculture of Escherichia coli strains for the biosynthesis of resveratrol. Microb Cell Fact. 2016;15:163.

28. Johnston TG, Yuan S-F, Wagner JM, Yi X, Saha A, Smith P, Nelson A, Alper HS. Compartmentalized microbes and co-cultures in hydrogels for ondemand bioproduction and preservation. Nat Commun. 2020;1 1:563.

29. Santos CNS, Koffas M, Stephanopoulos G. Optimization of a heterologous pathway for the production of flavonoids from glucose. Metab Eng. 2011:13:392-400.
30. Zhou S, Lyu Y, Li H, Koffas MAG, Zhou J. Fine-tuning the (2S)-naringenin synthetic pathway using an iterative high-throughput balancing strategy. Biotechnol Bioeng. 2019;116:1392-404.

31. Zhang W, Liu H, Li X, Liu D, Dong X-T, Li F-F, Wang E-X, Li B-Z, Yuan Y-J. Production of naringenin from D-xylose with co-culture of E. coli and $S$. cerevisia. Eng Life Sci. 2017;17:1021-9.

32. Yuan S-F, Hsu T-C, Wang C-A, Jang M-F, Kuo Y-C, Alper HS, Guo G-L, Hwang W-S. Production of optically pure I(+)-lactic acid from waste plywood chips using an isolated thermotolerant Enterococcus faecalis SI at a pilot scale. J Ind Microbiol Biotechnol. 2018;45:961-70.

33. Camus C, Ballerino P, Delgado R, Olivera-Nappa Á, Leyton C, Buschmann AH. Scaling up bioethanol production from the farmed brown macroalga Macrocystis pyrifera in Chile. Biofuels, Bioprod Biorefin. 2016;10:673-85.

34. Klöckner W, Büchs J. Advances in shaking technologies. Trends Biotechnol. 2012;30:307-14.

35. Suresh S, Srivastava VC, Mishra IM. Critical analysis of engineering aspects of shaken flask bioreactors. Crit Rev Biotechnol. 2009;29:255-78.

36. Vestergaard $\mathrm{M}$, Ingmer $\mathrm{H}$. Antibacterial and antifungal properties of resveratrol. Int J Antimicrob Agents. 2019;53:716-23.

37. Hwang D, Lim Y-H. Resveratrol antibacterial activity against Escherichia coli is mediated by Z-ring formation inhibition via suppression of FtsZ expression. Sci Rep. 2015;5:10029.

38. Westman JO, Franzén CJ. Current progress in high cell density yeast bioprocesses for bioethanol production. Biotechnol J. 2015;10:1185-95.

39. Yuan S-F, Guo G-L, Hwang W-S. Ethanol production from dilute-acid steam exploded lignocellulosic feedstocks using an isolated multistresstolerant Pichia kudriavzevii strain. Microb Biotechnol. 2017:10:1581-90.

40. Curcio MJ, Lutz S, Lesage P. The Ty1 LTR-retrotransposon of budding yeast, Saccharomyces cerevisiae. Microbiol Spectrum. 2015;3:1-35.

41. Zhou K, Qiao K, Edgar S, Stephanopoulos G. Distributing a metabolic pathway among a microbial consortium enhances production of natural products. Nat Biotechnol. 2015;33:377-83.

42. Dai Z, Huang M, Chen Y, Siewers V, Nielsen J. Global rewiring of cellular metabolism renders Saccharomyces cerevisiae Crabtree negative. Nat Commun. 2018:9:3059.

43. Francioso A, Mastromarino P, Restignoli R, Boffi A, d'Erme M, Mosca L. Improved Stability of trans-Resveratrol in Aqueous Solutions by Carboxymethylated (1,3/1,6)- $\beta$-d-Glucan. J Agri Food Chem. 2014;62:1520-5.

44. Thuan NH, Trung NT, Cuong NX, Van Cuong D, Van Quyen D, Malla S. Escherichia coli modular coculture system for resveratrol glucosides production. World J Microbiol Biotechnol. 2018;34:75.

45. Gibson DG, Young L, Chuang R-Y, Venter JC, Hutchison lii CA, Smith HO. Enzymatic assembly of DNA molecules up to several hundred kilobases. Nat Methods. 2009;6:343.

\section{Publisher's Note}

Springer Nature remains neutral with regard to jurisdictional claims in published maps and institutional affiliations.

Ready to submit your research? Choose BMC and benefit from:

- fast, convenient online submission

- thorough peer review by experienced researchers in your field

- rapid publication on acceptance

- support for research data, including large and complex data types

- gold Open Access which fosters wider collaboration and increased citations

- maximum visibility for your research: over 100M website views per year

At BMC, research is always in progress.

Learn more biomedcentral.com/submissions 